\title{
Allogeneic Bone Marrow Transplantation
}

National Cancer Institute

\section{Source}

National Cancer Institute. Allogeneic Bone Marrow Transplantation. NCI Thesaurus.

Code C15369.

A clinical treatment in which a patient receives bone marrow from a compatible, but not genetically identical, donor. 